\title{
Psychophysical and Neural Evidence for Emotion-Enhanced Perceptual Vividness
}

\author{
Rebecca M. Todd, ${ }^{1,2 \star}$ Deborah Talmi, ${ }^{3}$ Taylor W. Schmitz, ${ }^{1}$ Josh Susskind, ${ }^{4}$ and Adam K. Anderson ${ }^{1,2 \star}$ \\ ${ }^{1}$ Department of Psychology, University of Toronto, Toronto, Ontario M5S 3G3, Canada, ${ }^{2}$ Rotman Research Institute, Toronto, Ontario M6A 2E1, Canada, \\ ${ }^{3}$ School of Psychological Sciences, University of Manchester, Manchester M13 9PL, United Kingdom, and ${ }^{4}$ Institute for Telecommunications and \\ Information Technology, University of California, San Diego, San Diego, California 92093
}

\begin{abstract}
Highly emotional events are associated with vivid "flashbulb" memories. Here we examine whether the flashbulb metaphor characterizes a previously unknown emotion-enhanced vividness (EEV) during initial perceptual experience. Using a magnitude estimation procedure, human observers estimated the relative magnitude of visual noise overlaid on scenes. After controlling for computational metrics of objective visual salience, emotional salience was associated with decreased noise, or heightened perceptual vividness, demonstrating $\mathrm{EEV}$, which predicted later memory vividness. Event-related potentials revealed a posterior $\mathrm{P} 2$ component at $\sim 200 \mathrm{~ms}$ that was associated with both increased emotional salience and decreased objective noise levels, consistent with EEV. Blood oxygenation leveldependent response in the lateral occipital complex (LOC), insula, and amygdala predicted online EEV. The LOC and insula represented complimentary influences on EEV, with the amygdala statistically mediating both. These findings indicate that the metaphorical vivid light surrounding emotional memories is embodied directly in perceptual cortices during initial experience, supported by cortico-limbic interactions.
\end{abstract}

\section{Introduction}

Anecdotal and empirical evidence suggests that emotionally important events hold a special place in memory, where they are bestowed with a unique subjective vivid character. Here we propose that emotional events also evoke a previously unknown heightened subjective richness at the time they are experienced, rendering emotional events in the mind's eye with a special phenomenological character we call emotionally enhanced vividness (EEV).

Emotions are associated with the mutually enhancing effects of sympathetic arousal (Anderson et al., 2006b) and altered attention (Pessoa et al., 2002; Anderson et al., 2003; Talmi et al., 2008; Schmitz et al., 2009), which result in facilitated encoding of emotional events (Anderson and Phelps, 2001; Anderson, 2005; De Martino et al., 2009; Lim et al., 2009). It is well established that emotional events are more easily detected under impoverished conditions where attentional load is taxed (Soares and Ohman, 1993; Anderson, 2005), or stimuli are presented at the threshold of awareness (Nielsen and Sarason, 1981). Yet it remains unclear whether emotions change the subjective quality of above thresh-

Received Jan. 11, 2012; revised June 5, 2012; accepted June 28, 2012.

Author contributions: R.M.T., D.T., and A.K.A. designed research; R.M.T. performed research; J.S. contributed unpublished reagents/analytic tools; R.M.T. and T.W.S. analyzed data; R.M.T. and A.K.A. wrote the paper.

Funding for this project was provided by the U.S. National Institute for Mental Health and the Canadian Institutes of Health Research. We thank Tayler Eaton for her invaluable assistance with this project.

The authors declare no competing financial interests.

*R.M.T. and A.K.A. contributed equally to the writing of the paper.

Correspondence should be addressed to Dr. Rebecca M. Todd, Department of Psychology, University of Toronto, 100 St George Street, Toronto, 0N M5S3G3, Canada. E-mail: r.todd@acl.psych.toronto.edu.

DOI:10.1523/JNEUROSCI.0155-12.2012

Copyright $\odot 2012$ the authors $\quad 0270-6474 / 12 / 3211201-12 \$ 15.00 / 0$ old perception, resulting in a heightened subjective experience of perceptual vividness, or EEV.

Suggestive data from neuroscience indicate that emotional events may be endowed with a special quality of perceptual experience. Viewing emotionally salient images is associated with enhanced engagement of striate (Bradley et al., 2003; Padmala and Pessoa, 2008) and extrastriate visual cortex (Lang et al., 1998; Bradley et al., 2003; Vuilleumier et al., 2004; Sabatinelli et al., 2005), with augmented electrophysiological activity at relatively early latencies (Schupp et al., 2003). Yet the precise functional correlates of this visual cortex response remains unknown. Here we propose that increased visual cortex activation accompanying emotional events reflects the subjective impression of greater perceptual vividness.

To quantify perceptual vividness we adapted a magnitude estimation paradigm designed to measure subjective estimates of graded magnitudes of sensory stimuli (Stevens, 1957). In our noise estimation (NE) task, emotionally salient and neutral scenes were overlaid with sparse "visual noise." Participants were asked to estimate the relative degree of "noisiness" of each picture (Fig. 1). This provided a psychophysical index of whether greater emotional salience results in greater perceptual vividness, or EEV.

In addition to the magnitude of physical noise on the image, perceptual vividness is related to perceptual features, or the objective salience (contrast, color, complexity etc.) of an image. Here we used computational metrics of bottom-up image salience to control for the influence of objective salience on perceptual and mnemonic vividness. We further used eye movement monitoring to examine whether EEV was related to emotional influences on foveation. To examine whether emotions alter how 
we see rather than how we think, we examined the time course of cortical electrophysiological activity associated with emotional salience and objective noise. Finally, to examine the cortical sites and potential modulatory sources of EEV we used functional magnetic resonance imaging (fMRI). Assessing a direct brainbehavior relationship, we examined whether psychophysical judgments of EEV are supported by the visual cortices in concert with mesial regions associated with emotional salience (Lang et al., 1998; Bradley et al., 2003).

\section{Materials and Methods Materials}

All studies

25 negative photos and 25 positive photos were taken from the International Affective Picture System (IAPS). Twenty-five neutral photos were retrieved from the internet as well as the IAPS. Positive, negative and neutral images were selected to be equivalent on basic low level image statistics, equated in log luminance, $F_{(2,72)}<1$, and root mean square contrast, $F_{(2,72)}<1$. Positive and negative images were selected to be equivalent in ratings of arousal, or emotional salience. Based on ratings by a separate set of participants, negative, positive and neutral images did not differ in whether they contained single vs multiple objects, $F_{(2,72)}<1, p>0.1$, difficulty of figure ground discrimination (scale of $1-7), F_{(2,72)}<1, p>0.5$, scene complexity (scale of $1-7), F_{(2,72)}<1, p=0.5$, or number of human figures, $F_{(2,72)}<1, p>0.6$.

One of three levels of Gaussian all-color noise (less than standard, the same as standard, more than standard, which was 35\%, 45\%, 55\% in the NE experiment and Grayscale experiment, and 10\%, 15\%, 20\% in all subsequent experiments) was superimposed over each image using Adobe Photoshop 7.0. To minimize variance associated with differences in luminance and contrast across images, the "standard" image to be used for magnitude estimation was created to match each corresponding "comparison" image. Matched standards were created by applying a Gaussian kernel of 30-pixel radius to each image. In all studies except the electrophysiological and fMRI experiments, a square wave image composed of 7 equally spaced black bars was superimposed on the standards, providing edge contrast to enhance accurate noise assessment. Standards were all set to the intermediate level of noise density.

\section{Procedures}

\section{NE experiment}

We first examined whether emotionally salient events are associated with enhanced experience of perceptual vividness as measured by the NE procedure. Participants were 23 young adults (14 female, $18-25$ years), with normal or corrected to normal vision and no history of neurological problems, recruited from the University of Toronto campus. Two participants' data were excluded because average noise ratings were $>2.5$ SDs from the mean. Data from 21 participants ( 13 female) were used.

After informed consent and a brief practice session, participants performed the noise magnitude estimation task. A white fixation point was followed by the standard, shown for $2000 \mathrm{~ms}$, which was given the arbitrary modulus value of 100 . Immediately following, an image was presented for $2000 \mathrm{~ms}$ followed by a prompt requesting a numerical value for the magnitude of noise superimposed over the comparison image (Fig. 1). This focused on the perceptual scaling of the objective magnitude of noise, rather than the subjective quality of image clarity. Each image was repeated 3 times, once at each level of noise, and image presentation was randomized. After completing the NE task, participants rated each image for emotional salience by rating how "emotionally arousing" each image was. Each of the 75 photos was randomly presented without noise. Participants were asked to use a numerical scale from 1 (the image was not b

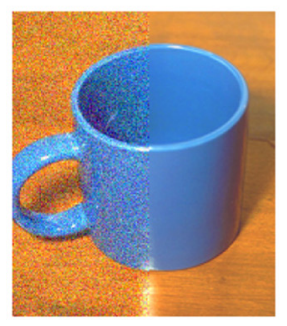

C

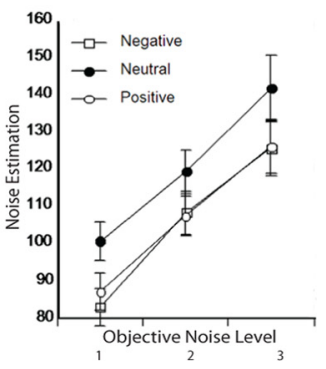

'emotionally arousing') to 7 (the image was extremely 'emotionally arousing').

For item analyses, each measure of interest was averaged across participants for each image used in the experiment. To obtain our emotional salience measure, we calculated the mean emotional arousal ratings for each image within participants and then averaged this value for each image across participants. To obtain a measure indexing perceptual vividness we calculated the inverse of the standardized $\mathrm{NE}\left(\mathrm{NE}^{-1}\right)$. As measures of objective salience we obtained several metrics for each image, including the number of edges (an aspect of scene complexity), hue, and a global computational metric of visual salience (Itti and Koch, 2001; Fig. $2 a)$. Objective image statistics were computed using the Image Processing Toolbox packaged with Matlab 7.0. Luminance statistics were derived from the average log luminance (Reinhard et al., 2002). Hue was calculated using Matlab's rgb2hsv function. Edges were detected using a Canny edge detector with a threshold of 0.5 . Lines were detected by using a Hough transform and the number of detected lines was calculated for each image. Visual salience has been defined as those basic visual properties, such as color, intensity, and orientation, that preferentially bias competition for rapid, bottom-up attentional selection (Itti and Koch, 2001). While visual salience has been used to predict sequential attentional selection of regions within a single image, we derived a measure of mean global saliency for each image to control for visual salience differences between images. Visual saliency was computed by averaging the saliency values across all image pixels using the Saliency Toolbox (Walther and Koch, 2006).

\section{Grayscale experiment}

In a follow-up control experiment we replicated the original NE findings when images were rendered in gray scale to control for potential influence of color on NE. Twenty-three young adults (13 females, 18-25 years) were tested. One participant's data were excluded due to average noise estimation ratings that were $2.5 \mathrm{SDs}$ above the mean. The materials and procedure were identical to the NE experiment with the exception that all stimuli were rendered in gray scale and emotional salience ratings were not collected.

\section{Sparse noise experiment}

Here we used sparser levels of noise density, controlling for the possibility that increased perceptual vividness for emotionally salient images was due to possible effects of specific noise levels on image content recognition. In the same study, to demonstrate the results were not dependent on a specific method of response, participants were asked to use an ordinal rather than a ratio scale. Participants were 27 young adults ( 14 females, $18-24$ years). Images and procedures were those in the first experiment with two exceptions: (1) Instead of typing in a number to quantify the amount of noise of an image relative to the standard, participants used a 22 point sliding scale that ranged from "A lot less noisy" to "A lot more 
a

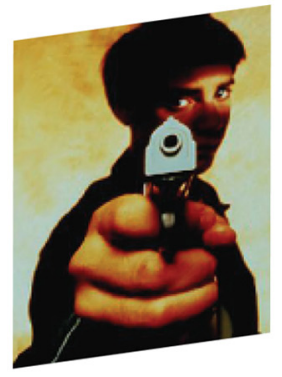

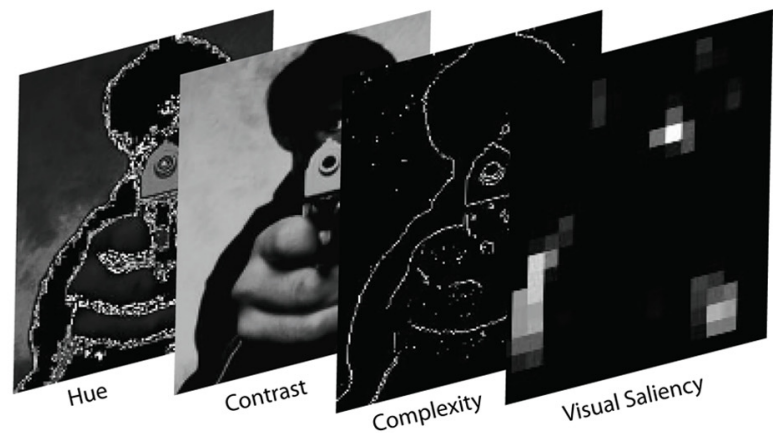

C

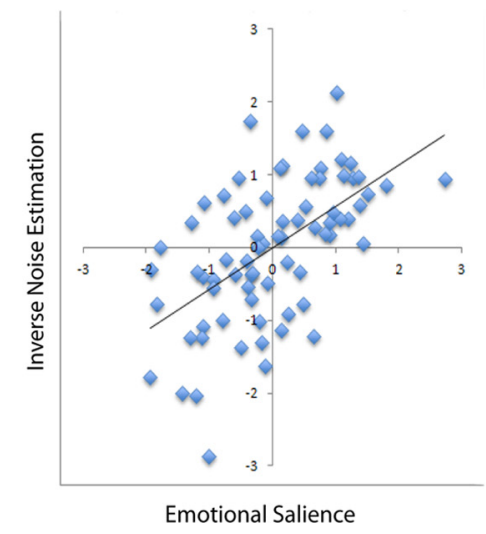

Emotional Salience b

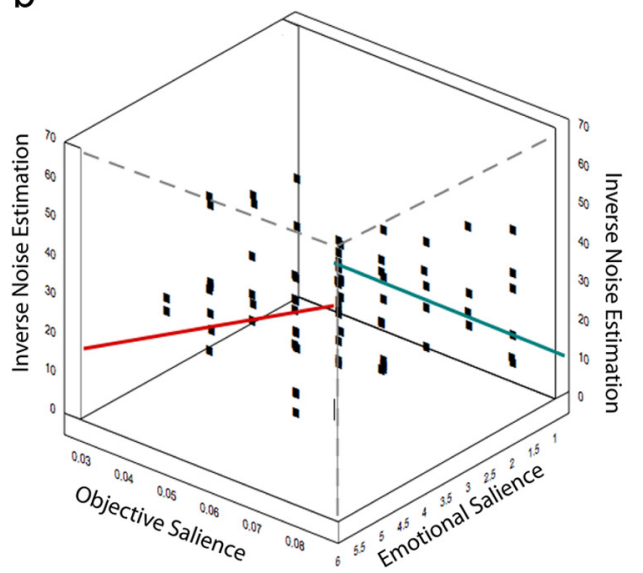

Figure 2. $\boldsymbol{a}$, For each image, images were rendered in grayscale and metrics were computed for hue, root mean square contrast, number of edges (scene complexity), and visual saliency. $\boldsymbol{b}$, Three-dimensional rendering of correlations between emotional
salience, visual saliency, and standardized inverse noise estimation ratings as a measure of perceptual vividness. $\boldsymbol{c}$, Correlation between emotional salience and ranked inverse standardized noise estimation measured after controlling for objective salience.

noisy," with "The same as standard" in the center of the scale. (2) Noise levels were $10 \%, 15 \%$, and $20 \%$, with the standard set always at $15 \%$.

\section{Single exposure experiment}

To control for potential effects of priming on NE ratings, each stimulus was presented only once in this version of the task. Twenty-six young adults ( $18-30$ years) participated. The NE task was identical to that used in the Sparse noise experiment except that each image was repeated only once at one of 3 levels of noise.

\section{Emotional salience control experiment}

A control experiment was performed to confirm that the level of overlaid noise did not influence ratings of emotional salience. Ten young adults ( 6 female) rated images for emotional salience at the lowest $(10 \%)$ and highest (20\%) levels of noise used in the majority of the NE tasks.

\section{Eye tracking experiment}

It is possible that altered foveation patterns associated with emotional images account for altered perceptual vividness. In a further control study, we measured number of eye movement fixations as a measure of overt attention. Twenty-four young adults (18-30 years) participated. One participant was excluded due to eye tracker calibration failure, and two withdrew from the study before completing the task, leaving 21 participants (17 female). The NE task was identical to that used in the Sparse noise experiment, with the following exception: To measure eye movement patterns in response to images when not obscured by noise, pictures were presented with $0 \%$, as well as at $10 \%$, and $20 \%$ noise. Standards were presented at $15 \%$ noise. Eye movements were measured with an Eyelink 1000 eye-tracking desktop monocular system (SR Research Ltd.) and sampled at a rate of $1000 \mathrm{~Hz}$. Participants sat $63 \mathrm{~cm}$ in front of the computer monitor and a chin rest was used to limit head movements. Standards and pictures were presented at a visual angle of $11.17^{\circ} \times 8.37^{\circ}$. A 9-point calibration was performed at the start of the experiment followed by a 0-point calibration accuracy test. Eye movement was analyzed using DataViewer software (SR Research Ltd.). The primary measure of interest was number of fixations per trial, which was used to quantify the amount of overt attention allocated to images in relation to noise judgment and emotional salience.

\section{Recognition memory study}

To investigate the link between perceptual vividness and memory vividness, participants $(N=27,14$ female) were asked to return a week after performing the Sparse Noise NE task and given a surprise recognition memory task. An additional set of images, 12 positive, 12 neutral, and 12 negative, were matched with the Session 1 images for emotional salience, scene content, contrast, and luminance. Participants were shown the original 75 images and the 36 new images. After rating an image as new, familiar, or recollected, participants rated the vividness of the memory for recognized items on a scale of $1-7$ ( $0=$ new image $)$ ranging from vague to detailed. Finally, participants rated all 111 images for emotional salience.

\section{Cued recall study}

To more closely approximate flashbulb memories and to control for the possibility that the relation between $\mathrm{NE}^{-1}$ and memory vividness in a recognition memory task reflects perceptual vividness when the stimulus is represented during test, we further conducted a cued recall study. The NE task was identical to that used in the Sparse noise experiment. Following completion of the NE task, 22 young adults ( 15 female) performed a spatial working memory task and filled out questionnaires. Forty-five minutes after completion of the NE task, participants were given a surprise cued recall task. Participants were given a list of one-or-two word cues (e.g., computer, dog, baby), each referring to a picture seen earlier in the task and instructed to describe the recalled image in writing in as much detail as possible. Each word cue was confirmed by 2 raters as providing an accurate description of the image described. Participants were instructed to describe the photograph in sufficient detail (e.g., objects, people, appearance, actions, place in the frame) that another person would be able to identify that exact image and distinguish it from other images seen in the task (Dolcos et al., 2004), and to include in the description any thoughts or emotions they recalled having had when viewing the image (Levine et al., 2002).

Two raters, blind to hypotheses, evaluated each image description using a reference set of images used in the task, and images whose description allowed for both identification and discrimination were classified as remembered. Descriptions that did not refer to an image in the set or that were a conflation of images were classified as not remembered. As an objective measure of memory detail, the number of details each remembered image was then scored according to a modified version of the Autobiographical Memory Scoring manual (Levine et al., 2002). Scoring was performed by a single rater, who was blind to our hypotheses, trained to reliability of $85 \%$ agreement against a "gold standard." Correct responses were scored for total number of details as well as for the number of thoughts/emotions recalled. Thoughts/emotions are details relating to the "mental state of the subject at the time of the event," including feeling states, thoughts, opinions, evaluations, or beliefs (Levine et al., 2002).

\section{Electrophysiological study}

We next used a version of the NE task using event-related potentials, or ERPs, to determine whether judgment of enhanced perceptual vividness is reflected in the amplitude of ERPs related to perceptual rather than 
conceptual processes. Data were analyzed from 31 young adults ( $13 \mathrm{fe}$ male) with normal or corrected to normal vision and no history of psychiatric disorders.

ERP data were collected in a version of the NE task identical to the Sparse noise experiment with the following exceptions. To have sufficient trials for ERP averaging in each image category, the level of noise on the pictures was held constant $(15 \%)$ and noise level was varied in the standards instead. Each image was seen 3 times paired with a standard at each of 3 noise levels $(10 \%, 15 \%$ and $20 \%)$. To create standards that varied as little as possible from paired images in featural characteristics, standards were created by phase scrambling each image and adding noise with no grating superimposed. A $1000 \mathrm{~ms}$ interstimulus interval was inserted between the offset of the standard and onset of the comparison image. Both standard and comparison images subtended a visual angle of $13^{\circ} \times 9.5^{\circ}$.

ERP data were collected using a 64-channel A.N.T. system and analyzed using EEProbe software (A.N.T. Software BV). Impedances were reduced to $<10 \mathrm{~K} \Omega$ before recording. All recordings were referenced to the right mastoid electrode. Signals were sampled at $512 \mathrm{~Hz}$. Individual EEG files were filtered using a finite impulse response filter using a 0.1-30 $\mathrm{Hz}$ bandpass. The continuous data were then re-referenced to the average of the two mastoids, and rejection markers were generated using channel thresholds (3 SDs over a sliding window of $200 \mathrm{~ms}$ ) over frontopolar channels FP1 and FP2 and occipital channels $\mathrm{O} 1$ and $\mathrm{O}_{2}$. Artifact-free trials were averaged for each subject from $100 \mathrm{~ms}$ before and $1000 \mathrm{~ms}$ following standard and picture onset, and baseline correction was performed by subtracting the mean of the $100 \mathrm{~ms}$ baseline window from the whole signal. Peak amplitudes were automatically extracted for P1 and P2 components from time windows based on grand mean data averaged across all conditions.

\section{Functional magnetic resonance imaging examinations}

Finally, we used fMRI to examine more precisely the cortical and subcortical substrates of perceptual vividness. Eighteen healthy young adult volunteers from Queen's University (18-30 years) with normal or corrected to normal vision and no history of psychiatric disorders participated in this study. Two participants were removed from subsequent fMRI analysis: one had excessive movement and one misunderstood the task, leaving 16 participants ( 10 female).

Localizer task. To localize category-selective regions of visual cortex, we used a block design task that alternated blocks of line-drawings of objects and scrambled line drawings to localize object-selective regions of the lateral occipital complex (LOC) - an early stage in the ventral object processing stream (Grill-Spector et al., 1998)—which we hypothesized would mediate emotional influences on the perception of objects. The task also included blocks of faces and places to localize other categoryselective regions of visual cortex. Each $20 \mathrm{~s}$ block contained 5 images presented for $4000 \mathrm{~ms}$ each. Blocks alternated randomly to minimize category predictability with 6 blocks of each image category. In each block participants performed a 1-back task.

Noise estimation task. fMRI data were collected during a variant of the NE ERP task where noise again was manipulated on the standard rather than comparison images to have sufficient trials for blood oxygenation level-dependent (BOLD) averaging in each stimulus category. Trials were presented in 5 separate runs of 30 trials. Each image was presented twice at 2 of 3 levels of standard noise (10\%, 15\% and 20\%) for a total of 150 trials ( 50 negative, 50 positive, and 50 neutral). In each $12 \mathrm{~s}$ trial a standard was presented for $1500 \mathrm{~ms}$, followed by a $500 \mathrm{~ms}$ interstimulus interval, followed by the picture which was presented for $1500 \mathrm{~ms}$. Both standard and comparison images subtended a visual angle of $13^{\circ} \times 9.5^{\circ}$.

After a randomly jittered interval of 1500,2000 , or $2500 \mathrm{~ms}$, the sliding scale response meter appeared for $4000 \mathrm{~ms}$, followed by a randomly jittered intertrial interval of 2000, 2500, or $3000 \mathrm{~ms}$. Fifty null trials (1/3), consisting of $12 \mathrm{~s}$ of fixation, were included at randomized intervals. After the scan, participants rated each image for emotional salience with the same procedure used in previous experiments.

fMRI acquisition. Imaging data were collected with a 3T Siemens scanner using a 12-channel head coil. Both the localizer and experimental tasks were programmed in E-prime Version 1.2 (Psychology Software
Tools). Stimuli were presented on a rear-mounted projection screen set at a resolution of $1024 \times 758$. For each subject, a three-dimensional magnetization prepared rapid-acquisition gradient echo pulse sequence was used to acquire a high-resolution T1-weighted structural volume: Repetition time $(\mathrm{TR})=1760 \mathrm{~ms}$; echo time $(\mathrm{TE})=2.2 \mathrm{~ms}$; field of view $($ FOV $)=256 \times 256$; slice thickness $=1 \mathrm{~mm} ; 176$ slices; total acquisition time $=7: 32 \mathrm{~min}$.

Three-dimensional (3D) field maps (coplanar with the fMRI slices) were acquired on each subject by measuring the phase of non-echoplanar imaging (EPI) gradient-echo images at two echo times (Jezzard and Balaban, 1995; Jenkinson, 2003). Parameters for the field mapping series were: $\mathrm{TR}=793 \mathrm{~ms}$; TE $1=5.19 \mathrm{~ms}$ and TE $2=7.65 \mathrm{~ms}$; flip angle $=60$; $\mathrm{FOV}=211 \mathrm{~mm}$. A total of 35 slices were acquired with a voxel size of $3.3 \times 3.3 \times 3.5 \mathrm{~mm}$. EPI parameters for the two functional tasks were: $\mathrm{TR}=2000 \mathrm{~ms} ; \mathrm{TE}=25 \mathrm{~ms}$; flip angle $=78^{\circ} ; \mathrm{FOV}=211 \mathrm{~mm}$.

Preprocessing. Functional activation was determined from the BOLD signal (Friston et al., 1995) using the software Statistical Parametric Mapping (SPM8, University College London, UK; http://www.fil.ion.ucl.ac. $\mathrm{uk} / \mathrm{spm} / \mathrm{software} / \mathrm{spm} 8$ ). We removed the first 5 time points from each functional run to allow for BOLD equilibration. After image reconstruction of the time series, we performed slice timing correction and motion correction using spatial realignment. We next applied the 3D field maps to unwarp the time series, thereby correcting for EPI image distortions caused by inhomogeneities in the magnetic field and subsequently coregistered individuals' time series with their T1 weighted structural image. The T1 image was bias-corrected and segmented using template (International Consortium for Brain Mapping) tissue probability maps for gray/white matter and CSF. Normalization parameters were obtained from the tissue segmentation procedure and subsequently applied to the time series data (resampling to $3 \mathrm{~mm}^{3}$ voxels). Finally, time series data were smoothed with a $6 \mathrm{~mm}$ full-width half-maximum Gaussian kernel.

First-level statistical models. For each subject, first-level general linear models were applied to localizer data and data from the NE task. Each model included within-session global scaling (default), high-pass filtering to remove low-frequency signal drift (period $=128 \mathrm{~s}$ ), and the AR1 method of estimating temporal autocorrelation.

For the localizer data, boxcar stimulus functions were convolved with the canonical hemodynamic response function (HRF). Conditionspecific regressors were included that modeled objects (line drawings) and scrambled objects as well as faces and places.

For the experimental data, a delta function regressor was modeled for image onset and convolved with the canonical HRF for each trial. We included 5 parametric modulators for image onsets, described by order of entry into the model as follows: (1) scene complexity, (2) hue, (3) contrast, (4) mean visual saliency, (5) $\mathrm{NE}^{-1}$. The fifth $\mathrm{NE}^{-1}$ regressor of interest was orthogonalized with respect to the objective salience regressors (1-4). Calculation of regressors 1-4 are described in previous sections of the paper. To create the $\mathrm{NE}^{-1}$ regressor, after averaging $\mathrm{NE}^{-1}$ across participants image by image as in previous item analyses, we calculated standardized residual $\mathrm{NE}^{-1}$ values after controlling for the objective noise level of the standard. That is, to probe brain activation specifically associated with the aspect of perceptual vividness linked to emotional salience, we removed the variance in $\mathrm{NE}^{-1}$ related to participant's (accurate) assessment of noise level relative to the standard to measure activation. The standardized residuals thus served as an index of $\mathrm{NE}^{-1}$ reflecting EEV, which we used for the first-level model.

Second-level statistical models. For the localizer task, $T$ contrast files for each condition (object drawings, scrambled objects, places and faces) from each individual were entered into a one-way ANOVA, with condition as the single factor. The contrast for [objects $>$ scrambled objects] was used to specify shape-selective activation in the LOC. Functionally defined masks were created using $10 \mathrm{~mm}$ spheres around maxima activations in the group maps $(51,-76,1$ and $-45,-79,1)$ thresholded at $p<0.05$ (familywise error, FWE). Anatomical masks for right and left amygdala and insula were created from automated anatomical labeling (AAL) templates (Tzourio-Mazoyer et al., 2002) based on a spatially normalized high resolution T1 single-subject dataset using the MaRSBAR toolbox (Brett et al., 2002). For the insula we further selected the dorsal granular subregion identified as primary interoceptive cortex 
Table 1. NE and Emotional Salience Means for all experiments

\begin{tabular}{|c|c|c|c|c|c|c|c|c|c|}
\hline \multirow[b]{2}{*}{ Experiment } & \multicolumn{3}{|c|}{ Emotional salience } & \multicolumn{3}{|c|}{ NE by objective noise } & \multicolumn{3}{|c|}{ NE by emotion category } \\
\hline & Negative & Positive & Neutral & Lower & Same & Higher & Negative & Positive & Neutral \\
\hline NE & 4.9 & 4.4 & 1.5 & 90.2 & 111.4 & 130.9 & 105.4 & 106.6 & 120.3 \\
\hline Grayscale & & & & 111.5 & 126.7 & 140.8 & 119.8 & 117 & 142.1 \\
\hline Sparse noise & 4.8 & 4.2 & 1.5 & 7.7 & 10.3 & 12.2 & 9.6 & 9.9 & 10.7 \\
\hline Single exposure & 4.2 & 3.8 & 1.3 & 9.8 & 11.7 & 13.3 & 11.5 & 11.5 & 12 \\
\hline Eye tracking & 4.5 & 4.2 & 1.8 & 5.8 & & 7.6 & 6.5 & 6.7 & 7 \\
\hline ERP & 4.5 & 4.2 & 1.5 & 12.2 & 11.6 & 11.1 & 11.3 & 11.4 & 12.1 \\
\hline fMRI & 4.3 & 4.0 & 1.4 & 8.2 & 7.5 & 6.7 & 7.3 & 7.5 & 7.8 \\
\hline
\end{tabular}

NE and Grayscale experiments: NE standard = 100; Sparse noise, Single exposure, and ERP studies: NE standard = 11 Eye tracking and fMRI studies: NE standard = 7.

(Craig, 2002) using anatomically based gray-matter regions of interest (ROIs) generated for a recent interoception study from our laboratory (Farb et al., 2012). To isolate primary interoceptive cortex within the insula, a mask of the long gyrus of the posterior insula, including gray matter only, was used using methods reported previously (Farb et al., 2012). Together, these ROIs were used for small volume correction for the NE task. Contrast files for $\mathrm{NE}^{-1}$ were subjected to a single-sample $t$ test at the group level. The initial SPM was thresholded at a height threshold of $p<0.005$, uncorrected, with a cluster extent threshold of 10 voxels (for rationale, see Lieberman and Cunningham, 2009). All results detected at this level are reported in Table 3 (see below).

Mediation analysis. A separate first level model, including regressors for each emotion category (positive, negative, and neutral) and standard noise level ( $10 \%, 15 \%$, and $20 \%$ noise), was used to extract activation for the mediation analysis. Stimulus onsets for emotion category and standard noise level were modeled using 6 separate delta function regressors. To characterize how the ROIs contribute to perceptual vividness, we extracted unadjusted signal from these regions for each participant, specifically the first eigenvariate of a $3 \mathrm{~mm}$ radius spherical volume of interest (VOI) centered on the coordinates of the peak activated voxel in each cluster. The first level contrast [emotionally salient $>$ neutral] was used to center the ROI spheres to specify regions implicated in emotional salience. ROI time series data excluded null trials. We transformed $\mathrm{NE}^{-1}$ values into an equivalent time series by convolving them with the hemodynamic response. As in other item analyses reported in this study, ROI time series and $\mathrm{NE}^{-1}$ ratings were averaged across participants-in this case at each time point rather than for each image. At the group level, regressions were run with ROIs as predictor variables and standardized residual $\mathrm{NE}^{-1}$ as the dependent variable. Regression and mediation analyses were performed in SPSS for alternate models using mediation syntax (Preacher and Hayes, 2004) and confirmed by bootstrapping (5000 samples).

Psychophysical interaction (PPI) analysis. The same first-level model was used as for the mediation analysis described above. The left and right amygdala were then used as seed regions for two psychophysiological interaction (PPI) analyses: In each subject, unadjusted activity was first extracted from volumes of interest (the first eigenvariate of a spherical VOI; $3 \mathrm{~mm}$ radius) centered on the coordinates of the left and right hemisphere amygdala respectively. Each PPI regressor was then calculated (using the SPM8 utility) as the volume-by-volume interaction between the deconvolved VOI time series and a binary vector coding for emotionally salient vs neutral trials. For each participant, the PPI, condition coding, and VOI regressors were then separately reconvolved with the canonical HRF and modeled with the same specifications as the above first-level SPMs. A positive contrast was specified for the interaction column, reflecting activations positively related to the PPI interaction term. At the group level contrast files were entered into a single sample $t$ test.

\section{Results}

In all analyses, reported $p$ values are Greenhouse-Geisser corrected when sphericity cannot be assumed and pairwise contrasts are Bonferroni corrected. Behavioral means for all studies are reported in Tables 1 and 2 . In the key experiments reported below, item analyses were used as a primary tool to probe percep-
Table 2. Memory means

\begin{tabular}{|c|c|c|c|c|}
\hline \multirow[b]{2}{*}{ Study } & \multicolumn{2}{|l|}{ Accuracy } & \multicolumn{2}{|c|}{$\begin{array}{l}\text { Memory } \\
\text { vividness measures }\end{array}$} \\
\hline & Arousing & Neutral & Arousing & Neutral \\
\hline Recognition memory & 0.67 & 0.71 & 3.65 & 3.4 \\
\hline Cued recall & 0.43 & 0.40 & 12.02 & 9.71 \\
\hline
\end{tabular}

Recognition memory accuracy: proportion of hits-false alarms. Cued recall accuracy: proportion of images correctly recalled. Recognition memory vividness: mean memory vividness ratings per image for correctly remembered images from 1 (vague) to 7 (vivid). Cued recall vividness: mean number of details remembered for each emotion category.

tual vividness in relation to the emotional salience of each image viewed in the task, while controlling for objective salience. For all item analyses-dependent measures of interest were averaged across participants for each image viewed in the task and subjected to regression analysis.

\section{Behavioral examinations}

\section{NE experiment}

NE data were submitted to a 2-way ANOVA with picture emotion (positive, negative, neutral) and noise (low, medium, high) as repeated measures. A significant effect of noise, $F_{(2,40)}=38.15$, $p<0.001$, revealed a linear increase in estimated magnitude with increasing objective noise, $F_{(1,40)}=76.24, p<0.001$. Each $22 \%$ increment in added Gaussian noise was associated with an approximate estimated $20 \%$ increase in NE, demonstrating that participants were highly accurate in reporting relative differences in the objective amount of noise. In addition to this sensitivity to objective noise, a significant main effect of emotion category, $F_{(2,40)}=11.23, p<0.001$, revealed that negative and positive images were judged to contain less noise relative to neutral, $F_{(1,40)}=22.34, p<0.001$ (Fig. $1 b$ ), and were judged on average as $15 \%$ less noisy, despite equal levels of objective noise. NE ratings for negative and positive images did not differ from each other, $F_{(1,40)}<1$ (Fig. $1 b$ ). Focused analyses examining the difference between emotional and neutral images at each level of objective noise showed that emotional images had lower NE at both low, $F_{(1,80)}=42.64, p<0.001$, and high levels of noise, $F_{(1,80)}=43.61, p<0.001$. Importantly, this decreased estimation of noise did not reflect decreased accuracy associated with distraction from the emotional content of the images since, as Figure $2 c$ indicates, participants were often more likely to overestimate objective noise levels of neutral items than emotionally salient items relative to standard. Emotionally salient images were perceived as containing less noise, consistent with our hypothesis that increased emotional salience would yield increased perceptual vividness.

To further evaluate the relation between emotional salience, objective salience and perceptual vividness as indexed by $\mathrm{NE}^{-1}$, we next used an item analysis. Correlational analysis revealed that subjective emotional salience was strongly correlated with $\mathrm{NE}^{-1}$, 
a

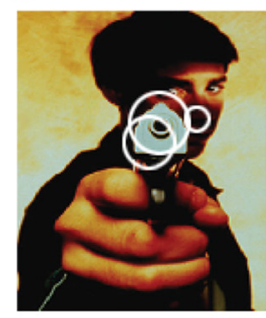

b

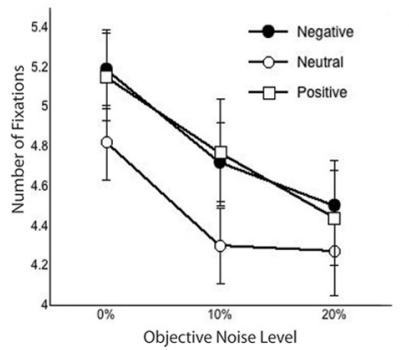

C

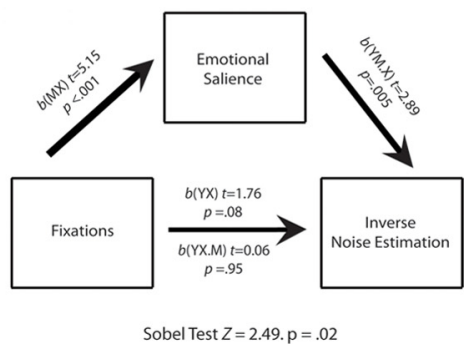

Figure 3. The role of eye movements in perceptual vividness. $\boldsymbol{a}$, Example of single subject's eye pattern fixations as an index of foveal sampling. $\boldsymbol{b}$, Mean number of fixations for positive, negative and neutral pictures at each level of objective noise. c, Mediation analysis showed that, after controlling for objective salience, the influence of foveal sampling on inverse noise estimation was mediated by emotional salience ratings, but not the reverse. [b(YX.M) designates the significance of number of fixations on perceptual vividness controlling for the mediator (emotional salience); $b(Y M . X)$ designates the significance of emotional salience on inverse noise estimation controlling for the predictor variable].

$r=0.62, p<0.001$ (Fig. $2 b$ ). $\mathrm{NE}^{-1}$ was also correlated with objective visual salience, $r=0.38, p=0.001$. Thus, with increasing emotional salience, images were perceived as higher in overall visual salience, and thus more vivid. We next performed a twolevel regression with all measures of objective salience included in the first level, and emotional salience in the second. After controlling for objective characteristics, there remained a highly systematic association, $R^{2}=0.45, R^{2} \Delta=0.26, p<0.001$, in which higher emotional salience was associated with greater $\mathrm{NE}^{-1}$ (Fig. $2 c$ ). Thus, despite their similar influence on $\mathrm{NE}^{-1}$, emotional and objective salience make dissociable contributions.

\section{Grayscale experiment}

We next replicated our NE results using grayscale images to control for the influence of color on perceptual vividness. A significant effect of noise, $F_{(2,42)}=10.74, p<0.001$, revealed a linear increase in estimated magnitude with increasing objective noise, $F_{(1,42)}=21.48, p<0.001$, with each $22 \%$ increment in added Gaussian noise associated with an approximate estimated $15 \%$ increase in estimated noise. A significant main effect of emotion, $F_{(2,42)}=5.86, p<0.01$, revealed that both negative and positive images were judged to contain less noise relative to neutral images, $F_{(1,42)}=11.60, p<0.005$, being judged on average $\sim 20 \%$ less noisy. There was no interaction between noise level and emotion category, $p>0.7$. Thus, the effect remained despite a dramatic change in the physical details of the images, suggesting that it is the emotional meaning of the image that resulted in altered NE.

\section{Sparse noise experiment}

We next replicated the results at sparser levels of noise. To minimize effects of noise level on image content recognition, pictures were presented at lower levels of noise than prior studies $(10 \%$, $15 \%$, and 20\%). Again, a significant effect of noise, $F_{(2,52)}=$ $89.08, p<0.001$, revealed a linear increase in NE with increasing objective noise, $F_{(1,26)}=90.05, p<0.001$. There was also a significant main effect of emotion, $F_{(2,52)}=32.73$, $p<0.001$, with emotionally salient images rated as more perceptually vivid than neutral, $F_{(1,26)}=59.46, p<0.001 . t$ tests revealed that while both negative and positive images were rated as less noisy than neutral images ( $p$ values $<0.001$ ) they did not differ in rated noisiness from each other $(p=0.21)$. Again, there was no interaction between noise level and emotion category, $p>0.7$. Thus, even with sparser noise levels we found high accuracy for objective NE, with lower NE indicating greater subjective perceptual vividness for emotionally salient images.

\section{Single repetition experiment}

To ensure that results were not due to differential effects arising from repetition of emotional and neutral images (Ishai et al., 2004), we replicated these results using a version of the task where each image was presented only once. NE results showed the typical main effect of noise level, $F_{(2,50)}=66.93, p<0.001$, with higher noise ratings for higher levels of noise, $F_{(1,25)}=76.32, p<$ 0.001 . Thus, even with decreased power associated with only one repetition of each image we found an effect of emotional salience, $F_{(2,50)}=4.95, p=0.01$, with lower noise ratings for emotionally salient than neutral images, $F_{(1,25)}=7.54, p=0.01$, suggesting that emotional modulation of NE was present upon first exposure to an image.

\section{Emotional salience control experiment}

Although the influence of emotional salience on $\mathrm{NE}^{-1}$ was significant at each level of noise, suggesting objective noise levels did not influence the impact of emotional salience on $\mathrm{NE}^{-1}$, we performed a further control task to ensure overlaid noise did not influence the ability to comprehend the emotional salience of the stimuli. A 2 (lowest noise, highest noise) $\times 3$ (emotion category) repeated-measures ANOVA revealed a significant effect of emotion category, $F_{(2,18)}=40.57, p<0.001$. Planned contrasts showed that positive and negative images were rated as more emotionally salient than neutral images at both highest and lowest levels of noise ( $p$ values $\leq 0.001$ ). Importantly, there was no main effect of noise level $(p>0.35)$ no emotion category by noise interaction $(p>0.2)$. Thus, the levels of noise did not have an impact on the subjective salience of emotional and neutral images.

\section{Eye tracking experiment}

Our results suggested that emotional salience reduces NE consistent with emotion-enhanced perceptual vividness. Rather than of central origin, reduced NE could reflect deployment of peripheral overt attention. In a further study we measured number of eye movement fixations as a measure of foveal sampling of images in relation to $\mathrm{NE}^{-1}$ and emotional salience. Across participants, there were more fixations associated with both lower objective noise levels, $F_{(2,40)}=44.28, p<0.001$, and more emotionally salient images, $F_{(2,40)}=5.44, p=0.02$, suggesting participants viewed emotionally salient images as if they were objectively less noisy (Fig. 3a). Mediation analysis based on an item analysis revealed that the influence of eye movement on perceptual vividness ratings is contingent upon the mediating influence of the emotional salience of the stimulus, $Z=2.49, p=$ 
a

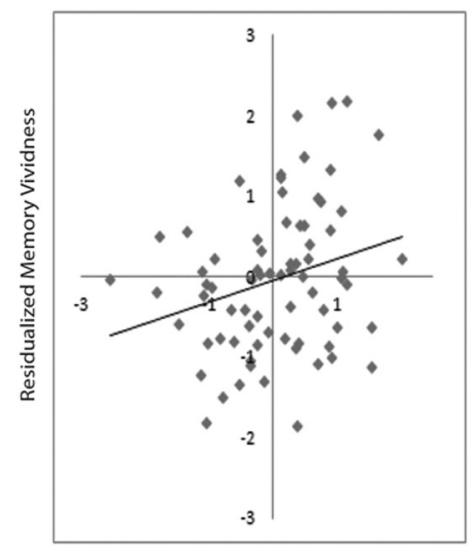

Residualized Inverse Noise Estimation b

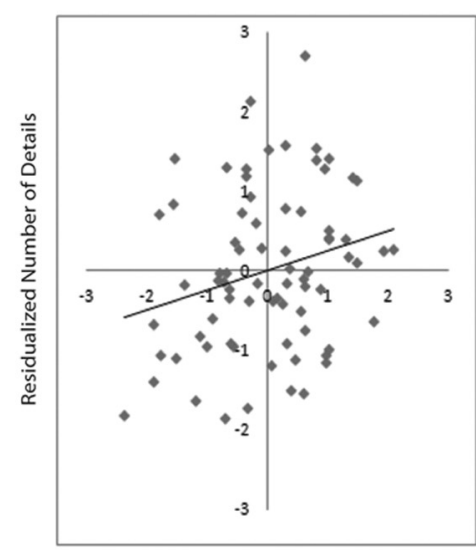

Residualized Inverse Noise Esimation
C

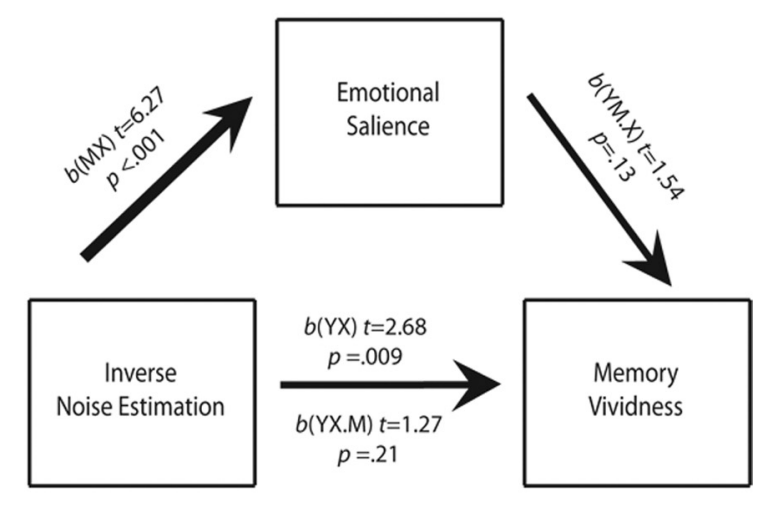

Sobel Test: $Z=1.48, p=.14$

Figure 4. Relation between perceptual vividness and measures of memory vividness. $\boldsymbol{a}$, Correlation between perceptual vividness and memory vividness in the recognition task after controlling for objective salience. $\boldsymbol{b}$, Correlation between inverse noise estimation and number of details recalled (a measure of memory vividness) in the cued recall task. $\boldsymbol{c}$, Mediation analysis showed that both emotional salience ratings and inverse noise estimation contributed to memory vividness ratings after controlling for objective salience. [b(YX.M) designates the significance of inverse noise estimation on memory vividness ratings controlling for the mediator (emotional salience); $b$ (YM.X) designates the significance of emotional salience on memory vividness controlling for the predictor variable].

0.02. In contrast, mediation analysis using emotional salience as the predictor and fixation count as the mediator revealed that fixation count did not mediate the effect of emotional salience on $\mathrm{NE}^{-1}, Z=0.06, p=0.95$. Thus, deployment of overt attention was correlated with-but did not account for-the way in which emotions alter perceptual vividness (Fig. 3c).

\section{Recognition memory study}

To investigate the link between perceptual and mnemonic vividness, we asked participants to return 1 week later for a surprise recognition memory task, in which they were shown previously seen images as well as unfamiliar images, matched with the original images for contrast and luminance as well as valence and emotional salience. Mean accuracy (hits-false alarms) and vividness (Mviv) ratings are reported in Table 2. There was no significant difference in corrected accuracy (hits-false alarms) or memory vividness ratings (Mviv) between emotionally salient and neutral trials ( $p$ values $>0.15$ ). We again used an item analysis to examine the extent to which, image by image, perceptual vividness and emotional salience predicted how vividly an image would be remembered. Recollection and Mviv were highly correlated, $r=0.79, p<0.001$, and we report results of Mviv, our primary metric of interest, for all correctly recognized trials. To ensure that our memory vividness measure was not driven by accuracy, Mviv was averaged across participants only for correctly remembered images. Emotional salience was associated with Mviv, $r=0.34, p=0.003$. Crucially, $\mathrm{NE}^{-1}$ was also correlated with Mviv, $r=0.35, p=0.002$. Data were next subjected to hierarchical regressions looking at effects of $\mathrm{NE}^{-1}$ and emotional salience on Mviv. To control for contributions of physical salience to memory vividness, measures of objective salience were entered at the first level. In two separate analyses, $\mathrm{NE}^{-1}$ and emotional salience were entered at the second level. $\mathrm{NE}^{-1}$ accounted for a significant portion of the variance in memory vividness after controlling for objective salience (Fig. $4 a$ ), $R^{2}=0.17$, $R^{2} \Delta=0.08, p=0.02$, as did emotional salience, $R^{2}=0.18$, $R^{2} \Delta=0.09, p=0.009$. To probe whether the influence of noise rating on Mviv was entirely mediated by emotional salience, we entered emotional salience and perceptual vividness into the same regression model and performed a Sobel test on memory vividness with $\mathrm{NE}^{-1}$ as the predictor and emotional salience as the mediating variable after controlling for objective salience (Preacher and Hayes, 2004). Here the mediation effect was not significant, $Z=1.46, p>0.1$, indicating that emotional salience did not fully account for the effect of $\mathrm{NE}^{-1}$ on memory, a result confirmed by bootstrapping (5000 samples) (Fig. 4c). We also tested the alternative model to see whether $\mathrm{NE}^{-1}$ mediated the influence of emotional salience on Mviv. This mediation effect was also not significant, $Z=1.14 p>0.1$, again confirmed by bootstrapping, suggesting $\mathrm{NE}^{-1}$ did not fully account for the influence of emotional salience on memory. In sum, emotionenhanced perceptual vividness contributes to recognition memory vividness.

\section{Cued recall study}

To ensure that memory vividness in the recognition memory task did not merely reflect the perceptual vividness when re-viewing the image, we conducted a cued recall study. Participants provided written descriptions of remembered images $45 \mathrm{~min}$ after completing the NE task. Mean accuracy and number of details are reported in Table 2. Cued recall results revealed no effects of emotional salience on memory accuracy. There was an effect of emotion on overall number of details remembered $F_{(2,46)}=7.71$, $p=0.001$, with more details remembered for emotionally salient than neutral images, $F_{(1,23)}=5.90, p=0.02$. We again used item analyses to probe effects of $\mathrm{NE}^{-1}$ and emotional salience on memory vividness measured (1) as number of details for correctly remembered items and (2) as number of thoughts and emotions - a subcategory of memory details associated with subjective feelings at the time of picture viewing. Again, $\mathrm{NE}^{-1}$ was strongly associated with emotional salience, $r=0.63, p<0.001$. Emotional salience was also associated with number of remembered details for each image, $r=0.46, p<0.001$ and number of thoughts/emotions, $r=0.41, p<0.001$. Crucially, $\mathrm{NE}^{-1}$ was correlated with remembered number of details $r=0.25, p=0.02$, as well as remembered thoughts and emotions $r=0.35, p=$ 0.002 . Moreover, after controlling for objective salience, $\mathrm{NE}^{-1}$ still accounted for variance in number of details $R^{2}=0.39$, 
a

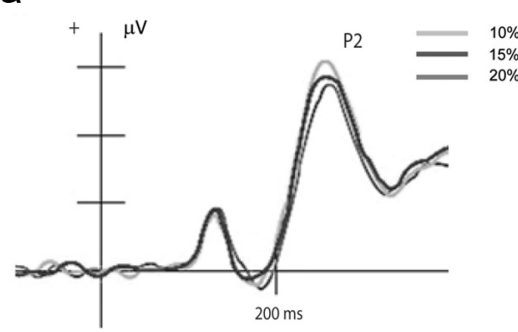

b

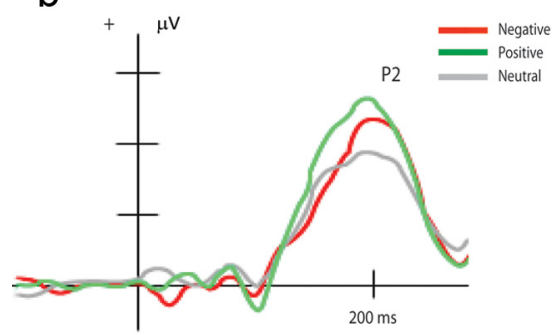

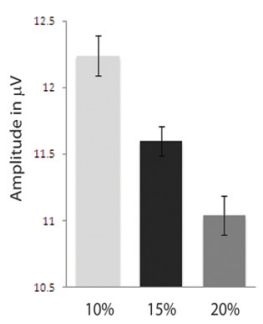

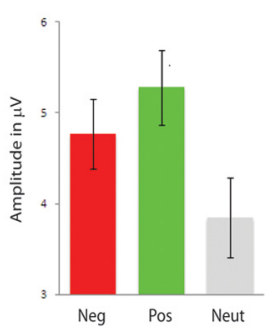

Figure 5. ERP correlates of noise and emotional salience. $\boldsymbol{a}$, ERP waves and P2 amplitudes following the standard at each level of objective noise demonstrate increasing amplitude with decreasing noise. $\boldsymbol{b}$, ERP difference waves (image minus scrambled standard) and P2 amplitudes showing greater amplitude for emotionally salient categories of image.

$R^{2} \Delta=0.04, p=0.04$, as well as number of thoughts/emotions $R^{2}=0.18, R^{2} \Delta=0.07, p=0.02$.

\section{Electrophysiological examinations}

We next used ERPs to determine whether the effect of emotional salience on cortical activation was similar to that of decreased levels of objective noise, and whether this common effect was reflected in the amplitude of ERPs related to perceptual (seeing) rather than conceptual (thinking) processes. Consistent with connectivity between visual cortex and motivation circuits (Amaral et al., 2003), we expected behavioral effects to be associated with ERPs linked to higher-order perceptual processes. Our focus was on the postsensory $\mathrm{P} 2$, an early-to-mid-latency positive ERP peak measured at occipitoparietal electrodes. The P2 has been implicated in object discrimination and enhanced attention to emotionally salient images (Carretié et al., 2004; Rousselet et al., 2008). We also examined the P1, an early positive peak associated with rapid, low-level sensory processing. Each ERP component's peak amplitudes were subjected to repeated-measures ANOVAs with emotion (positive, neutral, negative), laterality (right, central, left) and site (occipital, occipitoparietal) as within-subject factors.

To investigate cortical activation in response to objective noise independently of picture content, we first measured ERP amplitudes following presentation of the scrambled standard. For the $\mathrm{P} 1$, there was a noise level $\times$ laterality interaction $F_{(1,30)}=$ $4.27, p=0.01$, revealing higher amplitudes with greater levels of noise at right occipitoparietal sites. For the $\mathrm{P} 2$, a noise level $\times$ electrode site interaction, $F_{(2,60)}=3.83, p=0.03$, revealed a linear effect of noise level at occipital electrodes, $F_{(1,30)}=5.67$, $p=0.02$, with increasingly large amplitudes with decreasing levels of noise. In summary, over the right hemisphere, the P1 measuring sensory processes increased with objective noise level. In contrast, occipital P2 amplitude mirrored the perceptual vividness effect associated with emotional salience, with greatest amplitudes when noise levels were lowest (Fig. $5 a$ ). Thus, the P2,

associated with object processing and completion, was a reliable marker of objective levels of perceptual vividness.

To look at the effects of picture content while minimizing perceptual confounds, we subtracted ERP activation following picture presentation from activation following scrambled standard onset in each emotion condition. The first peak for this difference wave was at the latency of the P2 (208.34 ms) at occipitoparietal sites. There was no peak at the latency of the P1 (Fig. $5 b)$. Analysis of the $\mathrm{P} 2$ peak revealed a main effect of site, $F_{(2,60)}=$ $11.52, p=0.002$, with larger amplitudes at occipital sites. There was also a main effect of emotion, $F_{(2,60)}=6.02, p=0.004$, with higher amplitudes for emotionally salient than neutral images, $F_{(1,30)}=10.21, p=0.003$. Pairwise contrasts revealed that amplitudes were higher for negative $(p=0.04)$ and positive $(p=0.02)$ than neutral pictures, and did not differ between negative and positive $(p=0.22)$. Thus the $\mathrm{P} 2$, which peaked in amplitude over occipital complex by $210 \mathrm{~ms}$, and was greater for lower noise images, was also greater for emotionally salient images. Emotional salience augmented the salience of perceptual experience in a manner commensurate with the effect of decreasing levels of objective noise over occipital regions. Although indirectly related to NE, the P2 revealed that higher order occipital cortex responded to emotional stimuli as if they were embedded in lower noise, mirroring the psychophysical scaling of noise magnitude.

\section{Functional magnetic resonance imaging examinations}

While the ERP results suggest common timing and coarse cortical spatial distribution between decreased noise and emotional salience, it does not indicate whether these effects are related to NE, nor can it address the precise cortical and subcortical contributions to EEV. We next used fMRI to address the question of whether enhanced activation in the visual cortices, as well as other subcortical regions associated with the intensity of emotional experience, accounted for EEV. Parametric analyses were conducted to probe patterns of activation associated with $\mathrm{NE}^{-1}$ as a measure of EEV, on a trial-by-trial basis, after controlling for objective salience. Based on previous research (Bradley et al., 2003; Seeley et al., 2007) and our ERP findings, we used a priori masks to constrain our results in regions hypothesized to be involved in object (LOC) and affect (amygdala, insular regions associated with interoceptive sensation) perception. For each of these regions, we applied masks that were determined functionally using our independent localizer (LOC) or were anatomically derived from the AAL parcellation of the single subject MNI template brain (Tzourio-Mazoyer et al., 2002; amygdala, insula). These were used for small volume FWE corrected at $p<0.05$ to determine statistical significance. Small volume correction was performed in SPM by calculating thresholds correcting for the number of comparisons made, with the number of comparisons defined by the number of voxels in the prespecified masks (amygdala: 63 voxels; posterior insula: 37 voxels; LOC: 133 voxels). Results of the whole-brain analysis of regions modulated by $\mathrm{NE}^{-1}$ can also be found in Table 3 .

\section{Perceptual vividness}

Our primary goal was to investigate neural activation patterns underlying our main behavioral findings of EEV. After controlling for objective salience (hue, contrast, scene complexity, and mean visual saliency), activation in the left $\operatorname{LOC}(-51,-73,1$; $t_{(1,15)}=4.66$, FWE $p=0.008$, svc) and left amygdala $(-24,-4$, $-20 ; t_{(1,15)}=3.81$, FWE $p=0.02$, svc) as well as left dorsal posterior insula $\left(-36,-19,10 ; t_{(1,15)}=4.09, \operatorname{FWE} p=0.02, \mathrm{svc}\right)$ (Fig. 6a; for full uncorrected results, see Table 3) modulated 
Table 3. fMRI results from whole-brain analysis: regions parametrically modulated by perceptual vividness after controlling for objective salience

\begin{tabular}{lrrrllr}
\hline Brain region & $x$ & $y$ & $z$ & Voxels & $t$ & $p$ \\
\hline Left middle occipital & -51 & -73 & 1 & 64 & 4.66 & $<0.001$ \\
$\quad$ gyrus/BA 19 & & & & & & \\
Left insula/BA 48 & -36 & -19 & 10 & 28 & 4.09 & $<0.001$ \\
Left amygdala & -24 & -4 & -20 & 20 & 3.81 & 0.001 \\
Right insula/BA 48 & 36 & -10 & 19 & 19 & 3.70 & 0.001 \\
Left superior temporal & -51 & -7 & -5 & 36 & 3.44 & 0.002 \\
$\quad$ gyrus/BA 48 & & & & & & \\
\hline
\end{tabular}

The $x, y, z$ coordinates are in MNI space. $p$ values are uncorrected for multiple comparisons.

$\mathrm{NE}^{-1}$. These results suggest that EEV was associated with both object and emotional salience perception.

\section{Mediation analyses}

The above parametric analysis identified regions associated with EEV. However, to more directly examine the combined contributions of these regions to EEV we used statistical mediation analyses. In particular, we theorized that given its anatomical connectivity (Young et al., 1994) and importance in emotional and motivational salience (Cunningham et al., 2008), the amygdala would play a central role in mediating the relation between perceptual cortical recruitment and EEV.

First, to examine the relative influence of amygdala and LOC on $\mathrm{NE}^{-1}$, we performed a Sobel test on time series data (for details on time series extraction, see Materials and Methods). Left amygdala activation significantly mediated the effect of left LOC on $\mathrm{NE}^{-1}, Z=2.6, p=0.009$. Bootstrapping estimates (5000 samples) confirmed the effect within $95 \%$ confidence intervals. We also tested the alternative model to see whether left LOC activation mediated amygdala influence on $\mathrm{NE}^{-1}$. The mediation effect was not significant, $p>0.10$, again confirmed by bootstrapping, indicating that the LOC did not account for all of the influence of the left amygdala on perceptual vividness.

We next examined the mutual influences of posterior insula and amygdala activation on $\mathrm{NE}^{-1}$ ratings. Amygdala activation significantly mediated the effect of left insula on $\mathrm{NE}^{-1}, Z=2.53$, $p=0.01$, confirmed by bootstrapping (Fig. $6 c$ ). Insula activation did not mediate the influence of amygdala on $\mathrm{NE}^{-1}, p>0.10$. Thus, the amygdala mediated the relation between perceptual cortical responses - whether originating from external (LOC) or internal (posterior insula) experience-and the experience of EEV.

\section{PPI analyses}

Given results indicating a key role for the amygdala and LOC in perceptual vividness linked to emotional salience, we wanted to further examine whether emotional salience altered functional coupling between the amygdala and object-sensitive regions of the occipital cortex. In a final analysis, we examined psychophysiological interactions (PPI), separately using the time series from significant left and right amygdala voxels as seeds and emotionally salient (positive and negative) $>$ neutral trials as the condition coding. Significant PPIs were detected in the left LOC $(-39$, $-85,-2 ; t_{(1,15)}=4.78$, FWE $p=0.01$, svc) and right LOC (48, $-73,-2 ; t_{(1,15)}=6.31$, FWE $p=0.001$, svc), indicating that emotional salience modulated functional covariance between the left amygdala and LOC. Similarly for the right amygdala seed, significant PPIs were detected in the left $\left(-45,-85,-5 ; t_{(1,15)}=\right.$ 4.59$, FWE $p=0.01, \mathrm{svc})$ and right $\operatorname{LOC}\left(48,-73,-2 ; t_{(1,15)}=\right.$ 3.78 , FWE $p=0.03, \mathrm{svc}$ ) for emotionally salient relative to neutral trials. Thus, amygdala activation predicted LOC activation for emotionally salient but not for neutral images, suggesting that the coactivation between amygdala and LOC is enhanced by emotional salience.

\section{Discussion}

We demonstrate that the often-remembered vividness of emotional experience is embodied in a literal enhanced vividness of perceptual experience, or EEV, and its perceptual cortical expression. This association between emotional salience and perceptual vividness was demonstrated using the perception of noise in a quantitative psychophysical task. By having observers focus on the noise, rather than image content or signal, we could systematically evaluate perceived magnitude of an objective physical quantity while minimizing top down biases associated with evaluating image content. We further show that EEV predicts behavioral measures of subsequent memory vividness, both in recognition and recall. Consistent with the perceptual origin of EEV, ERPs over the occipital complex at just over $200 \mathrm{~ms}$ showed similar signatures for more emotionally salient images and for lower objective levels of noise. FMRI revealed that the LOC in the extrastriate cortex was sensitive to perceptual vividness, and that the amygdala was the modulatory source of EEV, mediating the influence of LOC on noise estimation. Thus, we show the oftenfound heightened activation in the visual cortices may support an increased subjective vividness of perceptual experience for emotional events.

Whereas previous studies have shown that emotionally salient images are more rapidly discriminated (Calvo et al., 2007), and are prioritized when attentional resources are limited (Anderson, 2005), our results demonstrate that the emotional salience of events is embodied directly in the subjective experience of visual clarity, endowing them with a special salience. While one interpretation of the data might be that participants are distracted by the emotional content and are thus less accurate at noise estimation, our results are not consistent with this interpretation. First, the ability to accurately scale increasing levels of noise was highly similar across image categories. Second, while viewing emotional pictures, observer's ratings often accurately matched the noise of the standard. In fact, as Figure $2 c$ indicates, participants were often more likely to overestimate objective noise levels of neutral items than emotionally salient items relative to standard. Thus, although of a different neural origin, the effect of EEV we report is similar to effects of attention, which has been shown to increase the resolution and visual signal-to-noise ratio of perception (Yeshurun and Carrasco, 1998). At low levels of noise, such as those used the present study, spatial attention has been found increase observer sensitivity to signal in low-level contrast discriminations (Lu and Dosher, 1998).

Here we found EEV engaged intermediate level visual object representations, specifically in the LOC, rather than earlier retinotopically mapped cortex, suggesting EEV primarily enhances signal at a higher level of object categorization. Within the LOC, attention and associated increased BOLD response have been associated with both increased gain and selectivity of shape selective neurons (Murray and Wojciulik, 2004). Emotional salience, and associated EEV, may not only amplify the neural response but also act to sharpen the population-level response to specific objects. This would create a more salient perception of the critical objects central to the emotional significance of a scene (Kensinger et al., 2011). Emotional salience has also been associated with increased temporal sampling (Droit-Volet and Meck, 2007), suggesting an additional potential mechanism underlying our findings of EEV. 
The influence of emotional salience on perceptual vividness was dissociable from objective salience and deployment of overt attention (eye movements), and the relation between overt attention and perceptual vividness was mediated by emotional salience. That is, altered foveation was not responsible for EEV; rather, it was accounted for by emotional salience, consistent with its central origin. Indeed, before the first eye movement, the P2 component associated with object processing, completion (Doniger et al., 2000; Rousselet et al., 2008), and salience (Straube and Fahle, 2010), was modulated by emotional salience. We further show the P2 was associated with both decreased objective noise levels and increased emotional salience in a manner consistent with the psychophysical scaling of emotional images as subjectively more vivid. Thus, cortical activation at the time of the P2 likely reflects the onset of a physiological correlate of the "flash" surrounding emotionally salient events. These data suggest this flash is unlike a spatial "spotlight." Rather, as a model of arousal-biased competition suggests (Mather and Sutherland, 2011), emotional salience appears to modulate object-based attention, enhancing the processing of objects that are subjectively salient to render them more physically salient in the mind's eye. The latency of this modulation is consistent with perceptual enhancement driven by extraction of image content-not too early, reflecting altered bottom up sensory processing, but not too late, reflecting only high level conceptual processing.

We used fMRI to examine specifically the relationship between mesial hubs of motivational neural circuits, a priori defined object-sensitive regions in the LOC, and their combined role in EEV. EEV parametrically varied with activation in the amygdala and the LOC, as well as in dorsal posterior insula. All of the regions activated by EEV fell within a well mapped motivational salience network (Bradley et al., 2003; Seeley et al., 2007). The amygdala sends projections to multiple levels of the ventral stream of the visual cortex, with increasing density of projections the further along the longitudinal fasciculus (Amaral et al., 2003), suggesting an important role in motivational influences on object perception and recognition. The amygdala has been associated with emotion-driven visual cortical activation (Lim et al., 2009) and enhanced encoding of emotionally salient stimuli under conditions of diminished attention (Anderson and Phelps, 2001; Vuilleumier et al., 2001; Lim et al., 2009)—although amygdala influences on early perceptual processes have been challenged (Todd and Anderson, 2009; Tsuchiya et al., 2009). The LOC region whose recruitment modulated perceptual vividness is associated with intermediate-level object processing (Grill-Spector et al., 1998; Kourtzi and Kanwisher, 2000; Op de Beeck et al., 2008). That the LOC was associated with EEV again suggests that EEV is related to the emotional salience of objects rather than the salience of low-level features. The amygdala is also reciprocally connected with posterior insular cortex (Mufson et al., 1981; Shi and Cassell, 1998). It has been suggested that, in primates, the posterior dorsal region of insula functions as primary interoceptive cortex, which is highly sensitive to attention (Farb et al., 2012), mediating a physiological sense of the whole body that is fundamental to emotional experience (Craig, 2002, 2009). Thus our results suggest that heightened perceptual vividness is unlikely to be purely visual, but may originate from heightened exteroceptive and interoceptive cortical engagement.

A further question centered on whether EEV was primarily due to the influence of extrastriate visual cortex on the amygdala or vice versa. Under conditions of reduced attention and awareness, amygdala activity may be driven by visual cortex activity (Lim et al., 2009). In contrast, under conditions approximating normal viewing, when attention is less challenged, the amygdala may drive enhanced visual processing via reentrant feedback to enhance the encoding of objects. Our formal mediation analyses of the brain-behavior correlations revealed that the relation between LOC activation and EEV was mediated by activity in the amygdala, rather than vice versa, suggesting a special role for motivational circuits in reentrant processes related to object recognition (Bar et al., 2006). The amygdala also accounted for the influence of posterior insula on EEV, potentially linking enhanced seeing with gut feeling (Barrett et al., 2007). PPI analysis further revealed that amygdala activation predicted LOC activation for emotionally salient but not for neutral trials. This finding is consistent with predictions made by models proposing that the amygdala influences visual cortex activation in a manner similar 
to- - but distinct from - the influence of frontoparietal networks supporting top-down attention (Pessoa, 2008; Cunningham and Brosch, 2012).

Indeed, such an influence of motivational circuitry on perceptual vividness is in contrast with engagement of lateral frontoparietal attention networks (Corbetta and Shulman, 2002). While the amygdala, LOC, and insula were associated with increased vividness, further exploratory analysis revealed that recruitment of the dorsolateral prefrontal cortex $\left(42,27,22, t_{(1,15)}=4.62, p<\right.$ 0.001 , uncorrected) and posterior parietal cortex $(57,-34,49$, $t_{(1,15)}=3.48, p<0.001$, uncorrected) was negatively correlated with perceptual vividness. We suggest that, rather than attending more during emotional events, individuals are attending differently, with less reliance on effortful attentional and executive processes to enhance encoding and memory. These emotional modulatory interactions (Kapp et al., 1994) may generate a unique aura of salience that influences the subjective experience of perceptual vividness in the moment (Berridge, 2003).

Our results showed that EEV predicted behavioral measures of subsequent memory measured by recognition memory vividness and number of details remembered in a cued recall task. Previous studies have shown that emotionally salient images are more likely to be remembered than neutral images (Cahill and McGaugh, 1998). In particular, emotionally salient images have been associated with greater memory for the central, emotionally salient, or goal-relevant elements of a scene (Kensinger et al., 2007a; Levine and Edelstein, 2009). Such findings have been associated with greater amygdala activation (Hamann et al., 1999; Canli et al., 2000; Kensinger et al., 2007b) as well as high-order visual cortex activation at encoding (Kensinger et al., 2007b; Talmi et al., 2008), and suggest that perceptual processing of salient events may be related to heightened memory (Kensinger et al., 2007b). The heightened vividness of emotional memories may be manifest in enhanced perceptual vividness during initial encoding. Here, although links between our own ERP and fMRI findings and memory vividness remain hypothetical, we provide novel evidence for a direct mapping between emotion-enhanced experience of perceptual clarity and behavioral measures of mnemonic vividness.

It is important to highlight that our results suggest enhanced perceptual vividness contributes to, but does not entirely account for, the heightened salience of emotional memories. We found that after controlling for its influence on perceptual vividness, emotional salience retains a unique contribution to memory vividness. Research in non-human animals (McGaugh et al., 2002) and humans (Nielson et al., 1996; Cahill and Alkire, 2003; Anderson et al., 2006a) also indicates that arousal induced after encoding influences memory consolidation to alter memory retention. Phasic physiological arousal at encoding may interact with more tonic arousal extending beyond initial encoding to alter memory consolidation (Cahill and Alkire, 2003). Thus, the influence of emotional salience and attendant arousal may occur at multiple timescales, which combine to endow emotional experiences and their associated memories with a uniquely vivid subjective character.

\section{References}

Amaral DG, Behniea H, Kelly JL (2003) Topographic organization of projections from the amygdala to the visual cortex in the macaque monkey. Neuroscience 118:1099-1120.

Anderson AK (2005) Affective influences on the attentional dynamics supporting awareness. J Exp Psychol Gen 134:258-281.

Anderson AK, Phelps EA (2001) Lesions of the human amygdala impair enhanced perception of emotionally salient events. Nature 411:305-309.
Anderson AK, Christoff K, Panitz D, De Rosa E, Gabrieli JD (2003) Neural correlates of the automatic processing of threat facial signals. J Neurosci 23:5627-5633.

Anderson AK, Wais PE, Gabrieli JD (2006a) Emotion enhances remembrance of neutral events past. Proc Natl Acad Sci U S A 103:1599-1604.

Anderson AK, Yamaguchi Y, Grabski W, Lacka D (2006b) Emotional memories are not all created equal: evidence for selective memory enhancement. Learn Mem 13:711-718.

Bar M, Kassam KS, Ghuman AS, Boshyan J, Schmid AM, Dale AM, Hämäläinen MS, Marinkovic K, Schacter DL, Rosen BR, Halgren E (2006) Topdown facilitation of visual recognition. Proc Natl Acad Sci U S A 103:449-454.

Barrett LF, Bliss-Moreau E, Duncan SL, Rauch SL, Wright CI (2007) The amygdala and the experience of affect. Soc Cogn Affect Neurosci 2:73-83.

Berridge KC (2003) Pleasures of the brain. Brain Cogn 52:106-128.

Bradley MM, Sabatinelli D, Lang PJ, Fitzsimmons JR, King W, Desai P (2003) Activation of the visual cortex in motivated attention. Behav Neurosci 117:369-380.

Brett M, Anton JL, Valabregue R, Poiine JB (2002) Region of interest analysis using an SPM toolbox [abstract]. In: 8th International Conference on Functional Mapping of the Human Brain. Sendai, Japan.

Cahill L, Alkire MT (2003) Epinephrine enhancement of human memory consolidation: interaction with arousal at encoding. Neurobiol Learn Mem 79:194-198.

Cahill L, McGaugh JL (1998) Mechanisms of emotional arousal and lasting declarative memory. Trends Neurosci 21:294-299.

Calvo MG, Nummenmaa L, Hyönä J (2007) Emotional and neutral scenes in competition: orienting, efficiency, and identification. Q J Exp Psychol (Hove) 60:1585-1593.

Canli T, Zhao Z, Brewer J, Gabrieli JD, Cahill L (2000) Event-related activation in the human amygdala associates with later memory for individual emotional experience. J Neurosci 20:RC99(1-5).

Carretié L, Hinojosa JA, Martín-Loeches M, Mercado F, Tapia M (2004) Automatic attention to emotional stimuli: neural correlates. Hum Brain Mapp 22:290-299.

Corbetta M, Shulman GL (2002) Control of goal-directed and stimulusdriven attention in the brain. Nat Rev Neurosci 3:201-215.

Craig AD (2002) How do you feel? Interoception: the sense of the physiological condition of the body. Nat Rev Neurosci 3:655-666.

Craig AD (2009) How do you feel—now? The anterior insula and human awareness. Nat Rev Neurosci 10:59-70.

Cunningham WA, Brosch T (2012) Motivational Salience: Amygdala tuning from traits, needs, values and goals. Curr Direct Psychol Sci 21:54-59.

Cunningham WA, Van Bavel JJ, Johnsen IR (2008) Affective flexibility: evaluative processing goals shape amygdala activity. Psychol Sci 19:152-160.

De Martino B, Kalisch R, Rees G, Dolan RJ (2009) Enhanced processing of threat stimuli under limited attentional resources. Cereb Cortex 19:127-133.

Dolcos F, LaBar KS, Cabeza R (2004) Interaction between the amygdala and the medial temporal lobe memory system predicts better memory for emotional events. Neuron 42:855-863.

Doniger GM, Foxe JJ, Murray MM, Higgins BA, Snodgrass JG, Schroeder CE, Javitt DC (2000) Activation timecourse of ventral visual stream objectrecognition areas: high density electrical mapping of perceptual closure processes. J Cogn Neurosci 12:615-621.

Droit-Volet S, Meck WH (2007) How emotions colour our perception of time. Trends Cogn Sci 11:504-513.

Farb NA, Segal ZV, Anderson AK (2012) Attentional modulation of primary interoceptive and exteroceptive cortices. Cereb Cortex. Advance online publication. Retrieved May 23, 2012. doi: 10.1093/cercor/bhr385.

Friston KJ, Holmes AP, Poline JB, Grasby PJ, Williams SC, Frackowiak RS, Turner R (1995) Analysis of fMRI time-series revisited. Neuroimage 2:45-53.

Grill-Spector K, Kushnir T, Hendler T, Edelman S, Itzchak Y, Malach R (1998) A sequence of object-processing stages revealed by fMRI in the human occipital lobe. Hum Brain Mapp 6:316-328.

Hamann SB, Ely TD, Grafton ST, Kilts CD (1999) Amygdala activity related to enhanced memory for pleasant and aversive stimuli. Nat Neurosci 2:289-293.

Ishai A, Pessoa L, Bikle PC, Ungerleider LG (2004) Repetition suppression 
of faces is modulated by emotion. Proc Natl Acad Sci USA 101:9827-9832.

Itti L, Koch C (2001) Computational modelling of visual attention. Nat Rev Neurosci 2:194-203.

Jenkinson M (2003) Fast, automated, N-dimensional phase-unwrapping algorithm. Magn Reson Med 49:193-197.

Jezzard P, Balaban RS (1995) Correction for geometric distortion in echo planar images from B0 field variations. Magn Reson Med 34:65-73.

Kapp BS, Supple WF Jr, Whalen PJ (1994) Effects of electrical stimulation of the amygdaloid central nucleus on neocortical arousal in the rabbit. Behav Neurosci 108:81-93.

Kensinger EA, Garoff-Eaton RJ, Schacter DL (2007a) Effects of emotion on memory specificity: memory trade-offs elicited by negative visually arousing stimuli. J Mem Lang 56:575-591.

Kensinger EA, Garoff-Eaton RJ, Schacter DL (2007b) How negative emotion enhances the visual specificity of a memory. J Cogn Neurosci 19:1872-1887.

Kensinger EA, Addis DR, Atapattu RK (2011) Amygdala activity at encoding corresponds with memory vividness and with memory for select episodic details. Neuropsychologia 49:663-673.

Kourtzi Z, Kanwisher N (2000) Cortical regions involved in perceiving object shape. J Neurosci 20:3310-3318.

Lang PJ, Bradley MM, Fitzsimmons JR, Cuthbert BN, Scott JD, Moulder B, Nangia V (1998) Emotional arousal and activation of the visual cortex: an fMRI analysis. Psychophysiology 35:199-210.

Levine B, Svoboda E, Hay JF, Winocur G, Moscovitch M (2002) Aging and autobiographical memory: dissociating episodic from semantic retrieval. Psychol Aging 17:677-689.

Levine LJ, Edelstein RS (2009) Emotion and memory narrowing: a review and goal relevance approach. Cogn Emot 23:833-875.

Lieberman MD, Cunningham WA (2009) Type I and Type II error concerns in fMRI research: re-balancing the scale. Soc Cogn Affect Neurosci 4:423-428.

Lim SL, Padmala S, Pessoa L (2009) Segregating the significant from the mundane on a moment-to-moment basis via direct and indirect amygdala contributions. Proc Natl Acad Sci U S A 106:16841-16846.

Lu ZL, Dosher BA (1998) External noise distinguishes attention mechanisms. Vis Res 38:1183-1198.

Mather M, Sutherland MR (2011) Arousal-biased competition in perception and memory. Perspect Psychol Sci 6:114-133.

McGaugh JL, McIntyre CK, Power AE (2002) Amygdala modulation of memory consolidation: interaction with other brain systems. Neurobiol Learn Mem 78:539-552.

Mufson EJ, Mesulam MM, Pandya DN (1981) Insular interconnections with the amygdala in the rhesus monkey. Neuroscience 6:1231-1248.

Murray SO, Wojciulik E (2004) Attention increases neural selectivity in the human lateral occipital complex. Nat Neurosci 7:70-74.

Nielsen SL, Sarason IG (1981) Emotion, personality, and selective attention. J Pers Soc Psychol 41:945-960.

Nielson KA, Radtke RC, Jensen RA (1996) Arousal-induced modulation of memory storage processes in humans. Neurobiol Learn Mem 66:133-142.

Op de Beeck HP, Haushofer J, Kanwisher NG (2008) Interpreting fMRI data: maps, modules and dimensions. Nat Rev Neurosci 9:123-135.

Padmala S, Pessoa L (2008) Affective learning enhances visual detection and responses in primary visual cortex. J Neurosci 28:6202-6210.
Pessoa L (2008) On the relationship between emotion and cognition. Nat Rev Neurosci 9:148-158.

Pessoa L, Kastner S, Ungerleider LG (2002) Attentional control of the processing of neural and emotional stimuli. Brain Res Cogn Brain Res 15:31-45.

Preacher KJ, Hayes AF (2004) SPSS and SAS procedures for estimating indirect effects in simple mediation models. Behav Res Methods Instrum Comput 36:717-731.

Reinhard E, Stark M, Shirley P, Ferwerda J (2002) Photographic tone reproduction for digital images. In: 29th Annual Conference on Computer Graphics and Interactive Techniques (SIGGRAPH), pp 267-276. ACM.

Rousselet GA, Husk JS, Bennett PJ, Sekuler AB (2008) Time course and robustness of ERP object and face differences. J Vis 8:3.1-3.18.

Sabatinelli D, Bradley MM, Fitzsimmons JR, Lang PJ (2005) Parallel amygdala and inferotemporal activation reflect emotional intensity and fear relevance. Neuroimage 24:1265-1270.

Schmitz TW, De Rosa E, Anderson AK (2009) Opposing influences of affective state valence on visual cortical encoding. J Neurosci 29:7199-7207.

Schupp HT, Junghöfer M, Weike AI, Hamm AO (2003) Emotional facilitation of sensory processing in the visual cortex. Psychol Sci 14:7-13.

Seeley WW, Menon V, Schatzberg AF, Keller J, Glover GH, Kenna H, Reiss AL, Greicius MD (2007) Dissociable intrinsic connectivity networks for salience processing and executive control. J Neurosci 27:2349-2356.

Shi CJ, Cassell MD (1998) Cortical, thalamic, and amygdaloid connections of the anterior and posterior insular cortices. J Comp Neurol 399: $440-468$.

Soares JJ, Ohman A (1993) Backward masking and skin conductance responses after conditioning to nonfeared but fear-relevant stimuli in fearful subjects. Psychophysiology 30:460-466.

Stevens SS (1957) On the psychophysical law. Psychol Rev 64:153-181.

Straube S, Fahle M (2010) The electrophysiological correlate of saliency: evidence from a figure-detection task. Brain Res 1307:89-102.

Talmi D, Anderson AK, Riggs L, Caplan JB, Moscovitch M (2008) Immediate memory consequences of the effect of emotion on attention to pictures. Learn Mem 15:172-182.

Todd RM, Anderson AK (2009) Six degrees of separation: the amygdala regulates social behavior and perception. Nat Neurosci 12:1217-1218.

Tsuchiya N, Moradi F, Felsen C, Yamazaki M, Adolphs R (2009) Intact rapid detection of fearful faces in the absence of the amygdala. Nat Neurosci 12:1224-1225.

Tzourio-Mazoyer N, Landeau B, Papathanassiou D, Crivello F, Etard O, Delcroix N, Mazoyer B, Joliot M (2002) Automated anatomical labeling of activations in SPM using a macroscopic anatomical parcellation of the MNI MRI single-subject brain. Neuroimage 15:273-289.

Vuilleumier P, Armony JL, Driver J, Dolan RJ (2001) Effects of attention and emotion on face processing in the human brain: an event-related fMRI study. Neuron 30:829-841.

Vuilleumier P, Richardson MP, Armony JL, Driver J, Dolan RJ (2004) Distant influences of amygdala lesion on visual cortical activation during emotional face processing. Nat Neurosci 7:1271-1278.

Walther D, Koch C (2006) Modeling attention to salient proto-objects. Neural Netw 19:1395-1407.

Yeshurun Y, Carrasco M (1998) Attention improves or impairs visual performance by enhancing spatial resolution. Nature 396:72-75.

Young MP, Scannell JW, Burns GA, Blakemore C (1994) Analysis of connectivity: neural systems in the cerebral cortex. Rev Neurosci 5:227-250. 